\title{
Quantum dots-in-a-well infrared photodetectors grown by MOCVD
}

\author{
Greg Jolley*, Lan Fu, H.Hoe Tan and C. Jagadish \\ Department of Electronic Materials Engineering \\ Research School of Physical Sciences and Engineering \\ The Australian National University, Canberra, A.C.T. 0200, Australia \\ *Email: gregory.jolley@anu.edu.au \\ N. Vukmirović and P. Harrison \\ School of Electronic and Electrical Engineering, \\ University of Leeds, Leeds LS2 9JT, United Kingdom
}

\begin{abstract}
The performance characteristics of InGaAs/GaAs quantum-dots-in-a-well infrared photodetectors grown by MOCVD are reported. A responsivity of $40 \mathrm{~mA} / \mathrm{W}$ at the peak detectivity of $3.2 \times 10^{9} \mathrm{cmHz}^{1 / 2} / \mathrm{W}$ has been achieved at a temperature of $77 \mathrm{~K}$. In an effort to understand the spectral behavior of the characterized devices band structure modeling has been performed.
\end{abstract}

Keywords-quantum dot in frared photodetector; Metal-Organic Chemical Vapor Deposition

\section{INTRODUCTION}

Infrared photodetectors with a spectral response in either the $3-5 \mu \mathrm{m}$ or $8-14 \mu \mathrm{m}$ atmospheric windows are utilized in numerous important applications including the thermal monitoring of industrial processes and medical diagnosis. Currently mercury cadmium telluride (MCT) is the material of choice for the development of IR photodetectors since sensitivity to any part of the IR band can be achieved by adjusting the mercury content (x) of $\mathrm{Cd}_{\mathrm{x}} \mathrm{Hg}_{(1-\mathrm{x})}$ Te. Also MCT detectors routinely demonstrate a very high level of performance [1].

However, MCT is a very difficult and expensive material to grow and process. Also the band gap of MCT is very sensitive to variations of the mercury content and therefore it is difficult to grow large areas of uniform MCT material suitable for the fabrication of focal plane arrays.

Motivated by the high cost of MCT detectors, intersubband quantum well infrared photodetectors (QWIPs) were developed as an alternative technology. The fabrication of QWIPs benefit from the well established III-V semiconductor growth and processing techniques. QWIPs are now a mature area of study and large uniform arrays of QWIPs are routinely fabricated.

However QWIPs suffer from some fundamental problems which have prevented them from becoming the dominant technology. Compared with MCT detectors, QWIPs have higher dark currents, lower responsivities and lower detectivities. QWIPs are insensitive to normal incident radiation, so additional fabrication steps are required to incorporate a grating.

Both MCT and QW IR photodetectors require cooling to very low temperatures to operate well. Such a cooling requirement can be a significant burden and adds to the overall cost of the photodetector system. In recent years there has been considerable interest in quantum dots (QDs) due to their unique fundamental properties as well as their potential use in superior optoelectronic devices.

Theoretical analysis has shown that quantum dot infrared photodetectors (QDIPs) can out perform QWIPs [2-4]. In particular it has been predicted that QDIPs can have lower dark currents and higher detectivities. Also the reduced phononelectron scattering rates of QDs can lead to devices with higher characteristic operating temperatures. QDIPs are inherently sensitive to normal incidence radiation eliminating the need of a grating.

However, for the theoretical predictions of QDIPs to be realized, multiple layers of densely packed QDs of high optical quality must be grown. In addition to this, the size and shape of the QDs has a strong influence on the QDIP performance characteristics.

In this study self-assembled QDs were grown in the Stranski-Krastanow (S-K) growth mode. Compared with quantum wells, the growth of self-assembled QDs is vastly more complicated. QD growth conditions are usually optimized to produce QDs of the highest optical quality and therefore it is an advantage if spectral tuning of QDIPs can be achieved without altering the QD growth parameters. The quantum dotsin-a-well (dwell) structure has been proposed as a means of achieving tuning without altering the dot growth conditions.

QDIPs with impressive performance characteristics have been reported in the literature [5-8]. However the far majority of these reported QDIPs have been grown by MBE. Due to the scalability of MOCVD, it is the industrial growth technique of choice. If QDIPs are to enjoy widespread use they must be developed by MOCVD. 


\section{EXPERIMENTAL DETAILS}

In this work $n-i-n$ InGaAs/GaAs dwell infrared photodetectors were grown by low pressure metal organic chemical vapor deposition on (001) oriented S.I. GaAs substrates. Figure 1 is a schematic of the structures grown. Growths began with the deposition of a $300 \mathrm{~nm}$ GaAs buffer layer followed by a $1 \mathrm{um} \mathrm{n}^{+} \mathrm{GaAs}$ bottom contact layer doped with $\mathrm{Si}\left(10^{18} \mathrm{~cm}^{-3}\right)$. The active region of all devices consists of $10 \mathrm{In}_{0.5} \mathrm{Ga}_{0.5}$ As QD layers embedded in an $\mathrm{In}_{0.15} \mathrm{Ga}_{0.85}$ As QW. Immediately after the deposition of the QD material on the bottom QW, a three second growth interrupt with As flow, was performed before the QDs were capped with the top part of the QW. Each Dwell region is separated by $50 \mathrm{~nm}$ of GaAs barrier material. After the active region was grown, an $\mathrm{n}^{+} 300 \mathrm{~nm}$ GaAs top contact layer doped with silicon $\left(10^{18} \mathrm{~cm}^{-3}\right)$ was deposited. To improve the quality of the barrier material, after depositing the initial 10nm of GaAs barrier material at the QD growth temperature, growth was halted while the temperature was ramped from $550^{\circ} \mathrm{C}$ to $600^{\circ} \mathrm{C}$ after which the remaining $40 \mathrm{~nm}$ of barrier material was deposited. The higher growth temperature improves the quality of the material and helped to re-planearize the growth surface before the next layer of dots is deposited.

It was found that the strain generated by 10 Dwell layers was adversely affecting the optical quality of the structure. To overcome this problem, the amount of dot material deposited was reduced and to compensate a growth interrupt was performed. The incorporation of a 3 second growth interrupt after the depo sition of the QDs was found to be a very effective way of maintaining a high QD density while reducing the accumulation of strain over a stacked structure.

$\left.\begin{array}{|c|}\hline \mathrm{GaAs}\left(\mathrm{n}=10^{18} \mathrm{~cm}^{-3}\right) 0.3 \mu \mathrm{m} @ 600^{\circ} \mathrm{C} \\ \hline \mathrm{GaAs} 400 \AA @ 600^{\circ} \mathrm{C} \\ \hline \mathrm{GaAs} 100 \AA @ 550^{\circ} \mathrm{C} \\ \hline \mathrm{In}_{0.15} \mathrm{Ga}_{0.85} \mathrm{As} 20-40 \AA @ 550^{\circ} \mathrm{C} \\ \hline 4.7 \mathrm{ML} \mathrm{In}_{0.5} \mathrm{Ga}_{0.5} \mathrm{As} \mathrm{QDs} @ 550^{\circ} \mathrm{C} \\ \hline \mathrm{In}_{0.15} \mathrm{Ga}_{0.85} \mathrm{As} 30 \AA @ 550^{\circ} \mathrm{C} \\ \hline \mathrm{GaAs}_{500 \AA @ 650^{\circ} \mathrm{C}}\end{array}\right\} \times 10$

Figure 1. Schemtic diagram of the device structure.

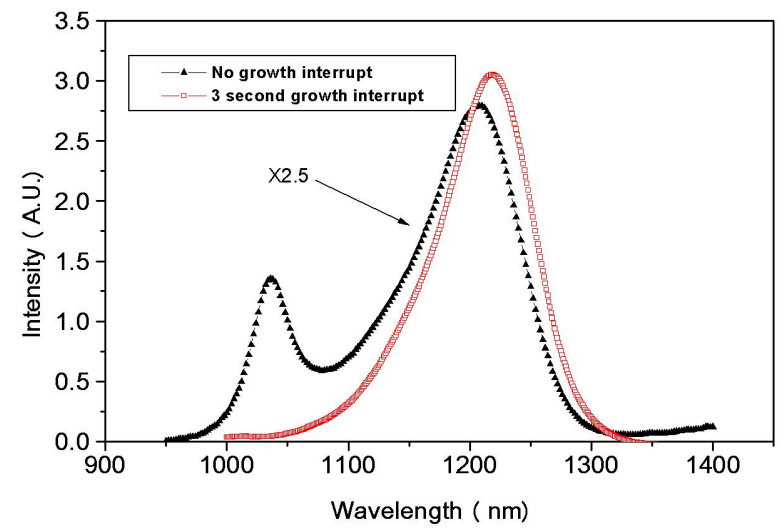

Figure 2. Room temperature photoluminescence of quantum dots formed with and without growth interrupts

The presence of the bottom InGaAs QW contributes to the formation of QDs during the growth interrupt. An increase of the dot density with the inclusion of a 3 second growth interrupt (GI) is evident from the room temperature photoluminescence results shown in figure 2. 4.7 ML of $\mathrm{In}_{0.5} \mathrm{Ga}_{0.5}$ As QD material was deposited on a $3 \mathrm{~nm}$ $\mathrm{In}_{0.15} \mathrm{Ga}_{0.85} \mathrm{As} \mathrm{QW}$. For one growth the dots were immediately capped with a similar $\operatorname{In}_{0.15} \mathrm{Ga}_{0.85} \mathrm{As} \mathrm{QW}$ and for the other, a three second growth interrupt was performed before the QDs were capped.

The wetting layer peak clearly diminishes when a growth interrupt is performed as more material from the wetting layer is encouraged to move into the quantum dots. Hence, the intensity of the QD peak increases significantly with growth interrupts.

Three structures that were identical apart from the thickness of the top QW were fabricated into devices. The thicknesses of the top QWs are as follows, device A 2nm, device B 3nm, and device C $4 \mathrm{~nm}$.

Part of the wafer used to fabricate device $\mathrm{C}$ was analyzed by transmission electron microscopy (TEM). From TEM results the QDs are estimated to have a height of about $4.5 \mathrm{~nm}$ and a base width of about $20 \mathrm{~nm}$ with a shape that can be represented by a truncated cone. These dot parameters were used as input for the band structure calculations.

Devices were fabricated into $250 \mu \mathrm{m}$ square mesa structures by standard lithography and wet chemical etching. Ohmic top and bottom contacts were formed by e-beam evaporation of $\mathrm{Ge} / \mathrm{Ni} / \mathrm{Au}$ followed by thermal annealing for 1 minute at $380^{\circ} \mathrm{C}$. Devices were then mounted into DIP packages and wire bonded for characterization.

The devices were placed into a Dewar and cooled by liquid nitrogen. Normal incidence spectral responses were obtained using a Nicolet Impact 400 Fourier Transform Infrared (FTIR) spectrometer and a Stanford Research Systems model SR570 low noise current preamplifier. 
Responsivity and detectivity measurements were obtained using a calibrated $800^{\circ} \mathrm{C}$ blackbody radiation source chopped at $160 \mathrm{~Hz}$ and a Stanford Research Systems model SR760 FFT spectrum analyzer. Dark current measurements were performed using a Hewlett Packard model 4140B pA meter and a DC voltage source.

\section{RESULTS AND DISCUSSION}

Devices were characterized for normal incidence spectral response to determine the amount of spectral tuning that occurred from width variations of the top QW. The spectral response of devices $A$ and $C$ are shown in figure 4 . The spectral response of device $\mathrm{B}$ is also essentially the same. Clearly the width of the top quantum wells has had very little influence on the spectral response of these detectors. There are a number of possible reasons for the lack of spectral tuning. The 8-band k.p technique incorporating the axial approximation was used to model the energy bands of the dwell structures and absorption coefficients were then calculated within the dipole approximation [9-10]. Strain effects were included in the modeling process by means of the finite element elastic continuum model. As has been previously reported [11], due to a large QD base to height ratio, modeling results reveals that significant absorption of in-plane (x-y) polarized light only occurs at longer wavelengths, in our case around $20 \mu \mathrm{m}$. Whereas peak absorption of $\mathrm{z}$-polarized light occurs at much shorter wavelengths, in our case around $8.2 \mu \mathrm{m}$ as seen in Fig. 3 (compared with the experimentally observed peak occurring at $6.5 \mu \mathrm{m})$. A spectral response at the longer wavelengths $(20 \mu \mathrm{m})$ has not been observed from these devices since the energy states that the electrons are being excited to by these wavelengths are too far below the continuum to contribute to photocurrent.

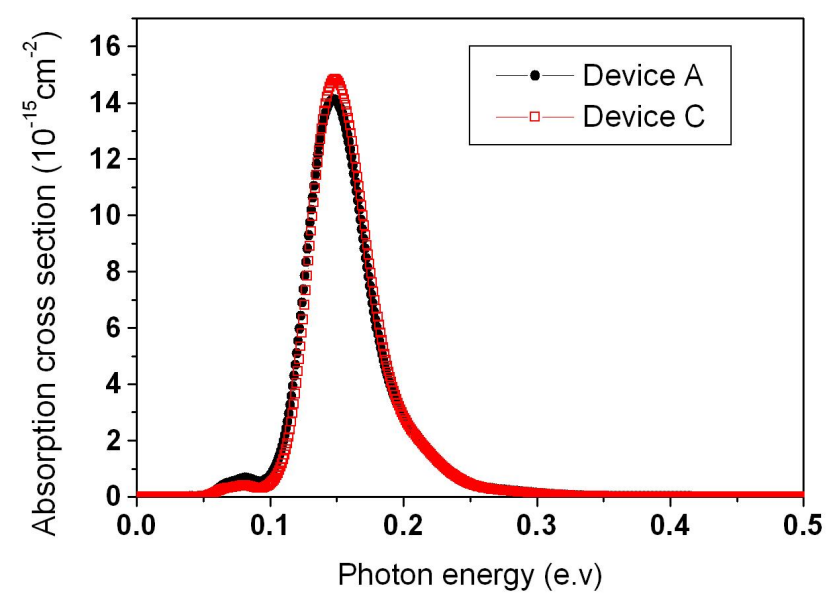

Figure 3. The calculated optical absorption cross section for QDs from devices $\mathrm{A}$ and $\mathrm{C}$.

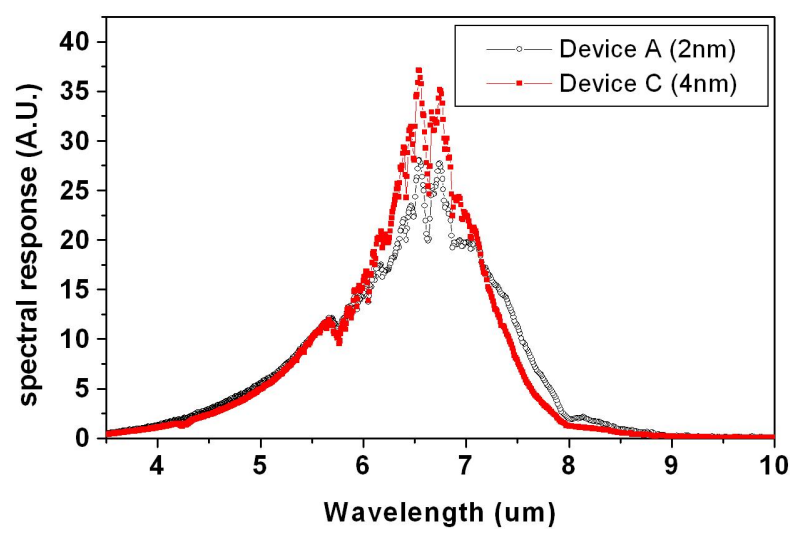

Figure 4. Normal incidence spectral response of photodetectors with different sized top quantum wells

In order for tuning of the $6.5 \mu \mathrm{m}$ spectral peak to be observed, it is expected that the first excited state, quantized in the z-direction, should lie within the energy range of the well. Therefore the lack of tuning could be attributed to the lack of a suitable first excited state. However simulation results suggest that even under favorable conditions tuning should be no greater than about $5 \mathrm{meV}$. Usually it is assumed that well width variations do not alter the quantum dot size or composition. In general this assumption cannot be guaranteed and any observed tuning could also be due to secondary effects such as a changing dot size.

The $77 \mathrm{~K}$ dark current-voltage characteristics of all dwell structures are essentially the same and are shown in Figure 5. The dark current density at $0.65 \mathrm{~V}$, which is the bias that gives the maximum detectivity, is $7.6 \times 10^{-5} \mathrm{Amps} / \mathrm{cm}^{2}$.

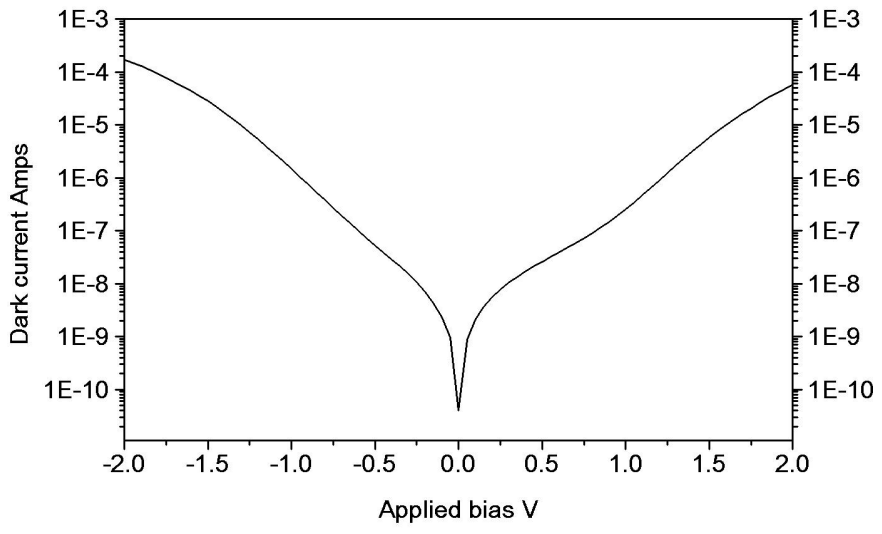

Figure 5. $77 \mathrm{~K}$ dark current- characteristics of device A 


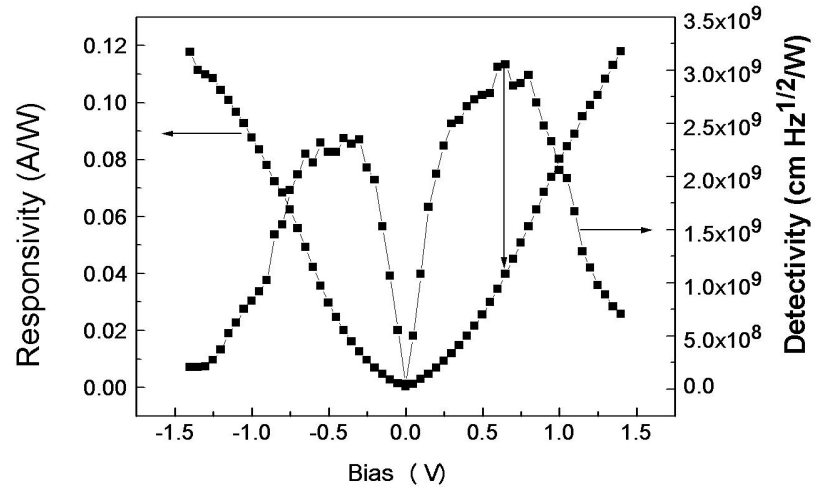

Figure 6. Normal incidence res ponsivity and detectivity at $77 \mathrm{~K}$

Normal incidence device responsivity and detectivity measured at $77 \mathrm{~K}$ is shown in figure 6 . The responsivity at the peak detectivity of $3.2 \times 10^{9} \mathrm{cmHz}^{1 / 2}$ is $40 \mathrm{~mA} / \mathrm{W}$. The small responsivity is partly attributed to the small absorption cross section of normal incidence radiation within the active wavelength range of the device.

\section{CONCLUSION}

In summary InGaAs/GaAs quantum dots-in-a-well infrared photodetectors with a responsivity of $40 \mathrm{~mA} / \mathrm{W}$ at the peak detectivity of $3.2 \times 10^{9} \mathrm{cmHz}^{1 / 2} / \mathrm{W}$ measured at $77 \mathrm{~K}$ have been grown by MOCVD. The performance characteristics of these devices are still far from the theoretically calculated potential characteristics of QDIP devices. To improve device performance, among other things, better control over dot size shape and density is required.

\section{ACKNOWLEDGMENT}

The authors would like to acknowledge the financial support of the Australian Research Council and the valuable technical assistance of Michael Aggett.

\section{REFERENCES}

[1] A. Rogalski, "Infrared detectors: status and trends," Progress in quantum electronics, vol. 27, pp. 59-210, 2003.

[2] V. Ryzhii, I. Khmyrova, M. Ryzhii and V. Mitin, "Comparison of dark current, responsivity and detectivity in different intersubband infrared photodetectors," Semicond. Sci. Technol. , vol. 19, pp. 8-16, 2004.

[3] J. Phillips, "Evaluation of the fundamental properties of quantum dot infrared photodetectors," J. Appl. Phys., vol. 91, pp. 4590-4594, 2002.

[4] M. Mashade 1, M. Ashry and A. Nasr, "Theoretical analysis of quantum dot infrared photodetectors," Semicond. Sci. Technol., vol. 18, pp. 891 900, 2003.

[5] L. Fu, P. Lever, K. Sears, H. H. Tan and C. Jagadish "InGaAs/GaAs quantum dot infrared photodetectors grown by metal-organic chemical vapor deposition," IEEE Electron device Lett., vol 26, pp. 628-630, 2005

[6] S. Krishna, D. Forman, S. Annamalai, P. Dowd, P. Varangis, T. Tumolillo, A. Gray, J. Zilko, K. Sun, M. Liu, J. Campbell and D. Carothers, "Demonstration of a $320 \times 256$ two-color focal plane array using InAs/InGaAs quantum dots in a well detectors", Appl. Phys. Lett., vol. 86, pp. 3501-3503, 2005.

[7] J. Liang Y. C. Chua, M. O. Manasreh, E. Marega and G. J. Salamo, "Braod-band photoresponse from InAs quantum dots embedded into InGaAs graded well," IEEE Electron device Lett., vol 26, pp. 631-633, 2005.

[8] S. Raghavan, P. Rotella, A. Stintz, B. Fuchs, S. Krishna, C. Morath, D A. Cardimona and S. W. Kennerly, "High-responsivity, normalincidence long-wave infrared, 7.2um $\mathrm{InAs} / \mathrm{In}_{0.15} \mathrm{Ga}_{0.85}$ As dots-in-a-well detector," Appl. Phys. Lett., vol. 81, pp. 1369-1371, 2002.

[9] Nenad Vukmirović, Zoran Ikonić, Vladimir D. Jovanović, Dragan Indjin and Paul Harrison, "Symmetry of k.p Hamiltonian in pyramidal InAs/GaAs quantum dots: Application to the calculation of electronic structure", Phys. Rev. B Vol. 72, 075356, 2005

[10] Nenad Vukmirović, Dragan Indjin, Zoran Ikonić, and Paul Harrison, "Origin of detection wavelength tuning in quantum dots-in-a-well infrared photodetectors", Appl. Phys. Lett., Vol. 88, 251107, 2006

[11] H.C. Liu, B. Aslan, M. Korkusinski, S.-J. Cheng, P. Hawrylak, "Detailed characterization of a systematic set of quantum dot infrared photodetectors," Infrared physics \& Technol., vol. 44, pp. 503-508, 2003. 